\title{
Follicular Variant of Papillary Carcinoma Thyroid with Massive Angioinvasion of the Internal Jugular Vein: Our Approach
}

\author{
Roshan Kumar Verma, Deepak Sarahan and Gautamjeet Raj Kumar \\ Department of Otolaryngology, Head and Neck Surgery, Postgraduate Institute of Medical Education and Research, Chandigarh, \\ India
}

Follicular variant of papillary carcinoma thyroid is an aggressive variant of papillary carcinoma thyroid. It is more commonly associated with extrathyroidal extension and regional lymphadenopathy. It can rarely be associated with microscopic vascular invasion but tumor thrombus into great veins is a rare phenomenon. We present a case of 60-year-old male with follicular variant of papillary carcinoma thyroid with tumor thrombosis in superior thyroid vein and internal jugular vein (IJV). We report a case of a 60-year-old male who presented with a large swelling in the lower part of left side of neck for 4 months. Clinical examination revealed a hard swelling of $12 \times 8 \mathrm{~cm}$ in left side of neck. Ultrasonography showed a solitary thyroid mass of the left lobe and a dilated left superior thyroid vein and ipsilateral IJV. Fine-needle aspiration cytology revealed follicular variant of PTC cells. Total thyroidectomy was done. A tumor thrombus was discovered in the superior thyroid vein and left IJV was found to be dilated. The left IJV with superior thyroid vein was ligated and excised. The patient recovered well after the operation with no local or distant metastasis detected. Follicular variant of PTC commonly spreads to the lymph nodes. Vascular spread via direct intravascular extension through superior thyroid vein is extremely rare. On palpation cord like IJV is felt on the involved side. Neck ultrasound play important role in the diagnosis. Aggressive surgical treatment with IJV ligation above and below the tumor thrombus is recommended to minimize the risk of potentially fatal complications of the intraluminal masses. Intravascular tumor extension into IJV of neck in follicular variant of PTC is rare and can be associated with serious consequences. Total thyroidectomy with thrombectomy with ligation of IJV must be done.

Key Words: Follicular variant of PTC, Superior thyroid vein thrombus, Internal jugular vein thrombus, Tumor thrombus

\section{Case Report}

A 60-year-old male patient presented to our tertiary care hospital with a history of swelling in front of left side of neck for four months. The swelling was initially of thumb size and progressively increased to size of big apple. The swelling was not associated with pain. There was no history of difficulty in swallowing, difficulty in respiration or recurrent cough. There was no features of hypothyroidism or hyperthyroidism.

Examination of the neck revealed a firm $12 \times 8 \mathrm{~cm}$ thyroid mass left to midline which was extending superiorly till upper border of thyroid cartilage, inferiorly till clavicle. Trachea was shifted to right side. A soft cystic swelling of $2 \times 2 \mathrm{~cm}$ was palpable just lateral to the upper left lobe of thyroid gland. No cervical lymph nodes were palpable in the neck. Fiber optic laryngeal examination revealed the bilateral mobile vocal cords.

Ultrasonography of the neck confirmed a $10 \times 6 \mathrm{~cm}$

Received September 9, 2020 / Revised October 8, 2020 / Accepted October 13, 2020

Correspondence: Roshan Kumar Verma, MD, PhD, Department of Otolaryngology, Head and Neck Surgery, Postgraduate Institute of Medical Education and Research, Chandigarh 160012, India

Tel: 91-7087009761, Fax: 91-172-2744401, E-mail: roshanverma@hotmail.com

Copyright (c) the Korean Thyroid Association. All rights reserved.

(c) (1) () This is an open-access article distributed under the terms of the Creative Commons Attribution Non-Commercial License (http://creative(1) (1) \& commons.org/licenses/by-nc/4.0/), which permits unrestricted non-commercial use, distribution, and reproduction in any medium, provided the original work is properly cited. 
solitary hypo echoic thyroid lesion involving the left lobe of the thyroid gland. Trachea was shifted to the right side. Left internal jugular vein (IJV) was dilated with thrombosis of the left IJV. Contrast enhanced computed tomography (CECT) of neck showed a well-defined, lobulated, heterogeneously enhancing mass lesion measuring $9.5 \times 7.2 \times 6.8 \mathrm{~cm}$ seen involving the left lobe of thyroid gland extending from $\mathrm{C} 3$ to D2 vertebral levels showing retrosternal extension (Fig. 1). CT angiography of neck was done which showed cluster of multiple prominent and tortuous vessels (vein) along the posterosuperior aspect of lesion. Superior thyroid vein of left side was dilated and tortuous. The left IJV was dilated and a breech was seen involving the medial wall of left IJV with extension of superolateral part of mass into the lumen of adjacent part of IJV. The intraluminal portion of the mass measured $31 \times 18 \mathrm{~mm}$ causing partial obstruction of left IJV. Right IJV was normal (Fig. 2).

Thyroid function tests were within normal limits (TSH 1.41 milli iu/ml, T3 $1.41 \mathrm{ng} / \mathrm{ml}$, T4 $8.06 .3 \mathrm{ug} / \mathrm{dl}$ ). Serum thyroglobulin was $1525 \mathrm{ng} / \mathrm{L}$ and serum PTH was $34.6 \mathrm{pg} / \mathrm{ml}$.

Fine-needle aspiration cytology of the thyroid nodule was done which was reported as follicular variant of papillary thyroid carcinoma.

Total thyroidectomy with left selective and central neck dissection was done. Superior thyroid vein was dilated and the tumor was found filling the superior thyroid vein and extending into the left IJV (Fig. 3). The left IJV was dilated and intraoperative palpation showed a $2 \times 3 \mathrm{~cm}$ hard intraluminal mass filling the IJV (Fig. 4). $5 \mathrm{~cm}$ segment of the IJV along with tumor thrombus was removed along with tumor. Longitudinal
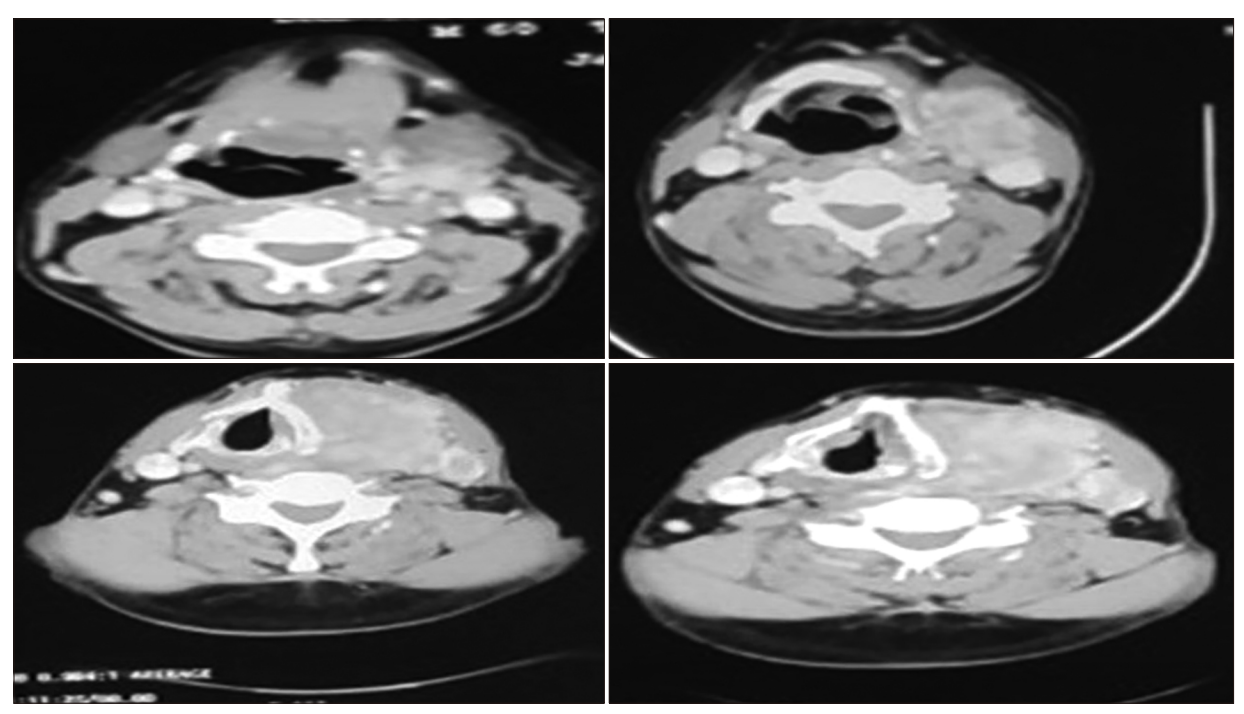

Fig. 1. Contrast enhanced computed tomography of the neck showing enlarged left lobe of thyroid and enhancing soft tissue mass filling and completely obliterating the internal jugular vein.
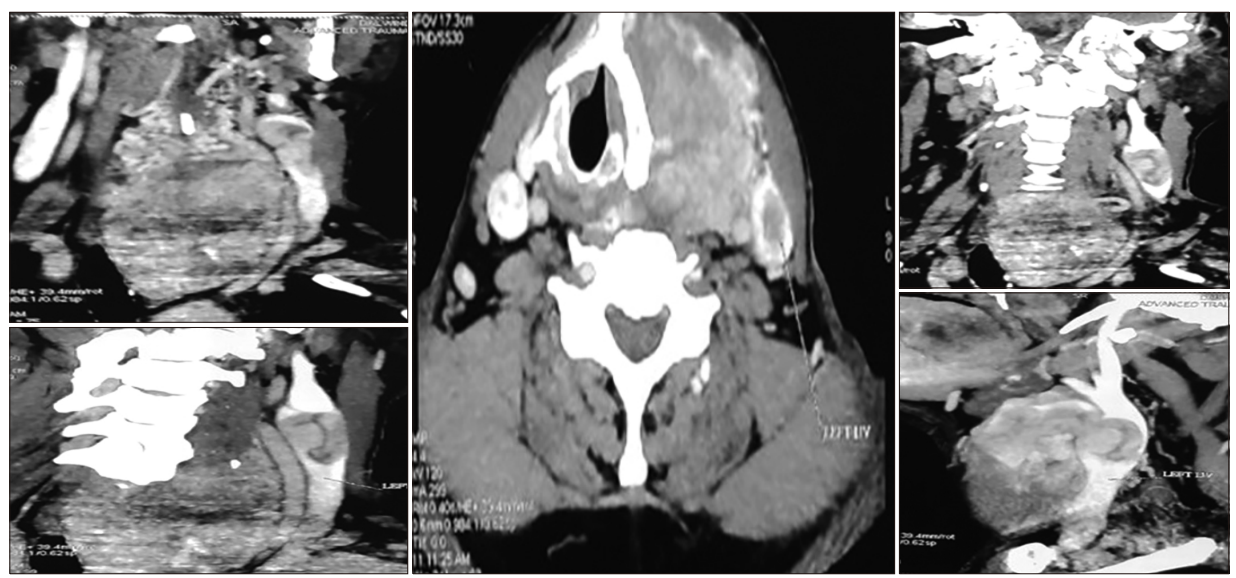

Fig. 2. CT angiography showing dilated and tortuous Internal jugular vein of left side with soft tissue mass filling the lumen of internal jugular vein. 

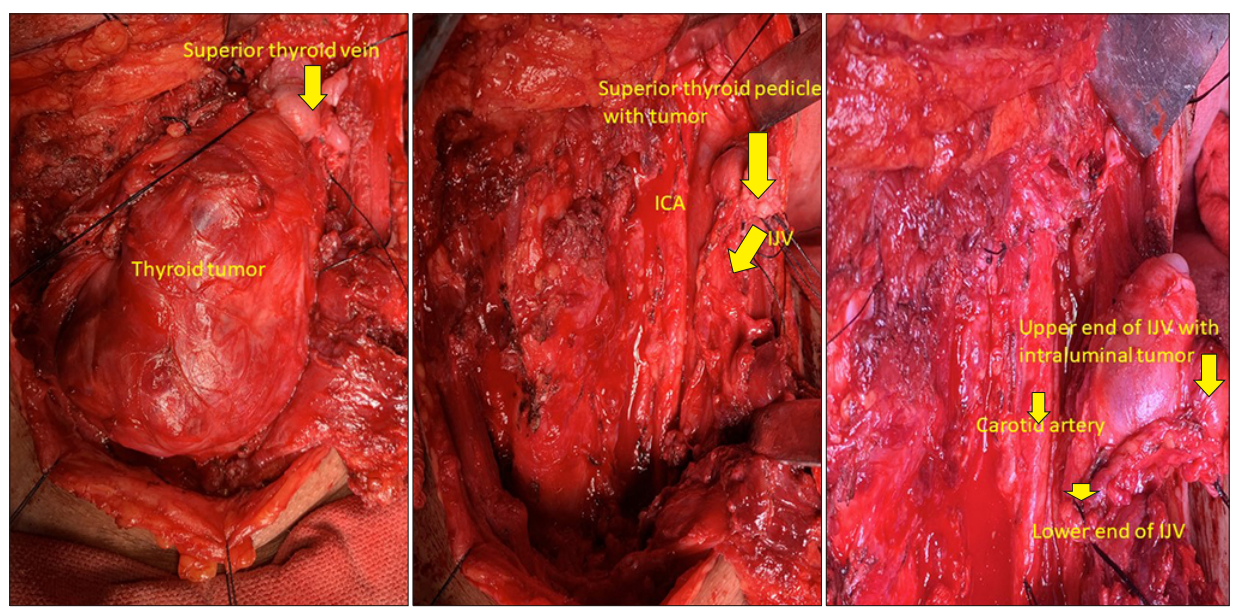

Fig. 3. Intraoperative photograph showing enlarged and tortuous left superior thyroid vein and soft tissue mass filling lumen of left superior thyroid vein extending into internal jugular vein.

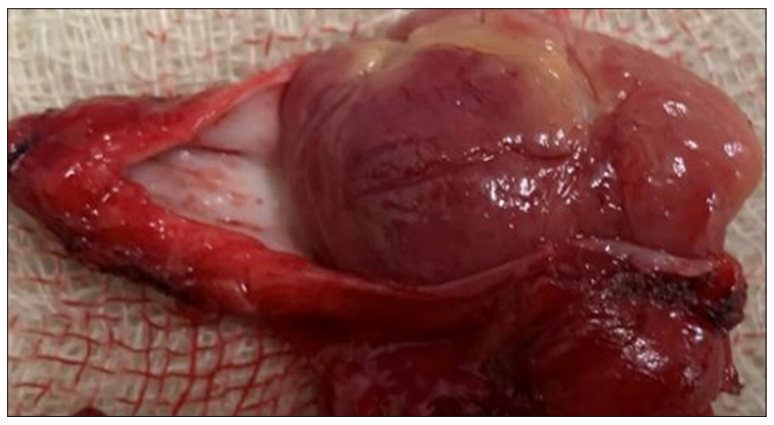

Fig. 4. Internal jugular vein opened from behind showing soft tissue tumor within its lumen.

venotomy revealed a tumor thrombus with endothelial adhesion (Fig. 2). Recovery was uneventful.

Histopathology the left thyroid mass and intraluminal mass in the IJV was reported as the follicular variant of papillary carcinoma thyroid. Radionuclide scan with Tc99m was done and it did not show any evidence of metastatic disease in any other part of the body.

\section{Discussion}

Follicular variant of papillary thyroid carcinoma is a histological variant of papillary carcinoma thyroid which has follicular architecture but nuclear features are classical of papillary carcinoma thyroid. ${ }^{1)}$ It is associated with a higher incidence of extrathyroidal tumor invasion and regional lymphadenopathy. ${ }^{2,3)}$ Incidence of distant metastasis is less than $1 \%$ at presentation.

Kaufmaan and Graham were the first to report two cases of intraluminal vascular tumor thrombosis in thyroid cancer. ${ }^{5,6)}$ Invasion/intraluminal spread into great vessels of the neck in differentiated thyroid carcinoma is extremely rare and reported incidence is $1.3 \%{ }^{7)}$ Vascular invasion is more common in follicular carcinoma of the thyroid as compared to papillary thyroid carcinoma.

Vascular invasion into IJV can occur commonly via extravascular luminal invasion by metastatic lymph node with extracapsular invasion or via direct infiltration by the primary tumor and the tumor is staged as T4b. This present case was an interesting as we saw intravascular extension of tumor cells by direct intraluminal invasion through superior thyroid vein extending into IJV downwards with intact venous walls. The primary tumor had no extracapsular invasion.

Clinical presentation of the thrombosis of IJV may vary from asymptomatic to acute pulmonary emboli to superior vena cava syndrome., ${ }^{8,9)}$ Our case was asymptomatic at presentation as tumor thrombus in IJV was only partially obstructing its lumen. Diagnosis of such type of tumor thrombi in the vascular lumen is difficult clinically in the absence of symptoms.

Preoperative ultrasound however can diagnose this condition. Doppler spectral analysis can provide information about the location, side, and degree of occlusion of the central vein lumen by assessing spectral waveforms of IJV on both sides. ${ }^{10)}$ However, ultrasound findings are operator dependent. CT and MRI are better modalities for diagnosing vascular spread. The CT and MR angiography will better delineate the tumor thrombi and extent of vascular involvement. In 
our case, CT angiography showed the embolus involving the left IJV.

Intraluminal invasion should be differentiated from the direct extravascular invasion which is more common in undifferentiated thyroid carcinoma. This differentiation will define the plan of management. In the intraluminal type of tumor invasion, the tumor mass in the vein is usually surrounded by a fibrous capsule which can sometimes be excised from the vessel wall and repaired." However, the segment of involved vessel has to be sacrificed if the tumor is adhered to vessel wall to get safe margins. While in extravascular invasion, the segment of vessel involved has to be sacrificed. In our case though the tumor was intraluminal, $5 \mathrm{~cm}$ segment of the IJV was resected as the tumor was completely filling the lumen of IJV and was adherent to its wall. ${ }^{11)}$

Nevertheless, any direct exposure of malignant cells to the circulation is an indicator of poor outcome with high probability of pulmonary and distant metastasis. ${ }^{12)}$ Due to the rarity of thyroid cancer vascular thrombi, no established management guidelines exist. Intraluminal extension is not a contraindication for aggressive surgical treatment in differentiated thyroid cancers due to the relatively good prognosis of PTC as well as to decrease the risk of SVC obstruction, sudden death from air-way occlusion, tumor embolism or fatal right atrial obstruction. ${ }^{6}$ The reported survival of patients with IJV invasion or other great cervical veins by thyroid cancer is $2-5$ years. ${ }^{13)}$

\section{Conclusion}

Intravascular tumor extension of PTC is rare with potential serious complications. Preoperative diagnosis using neck US is possible but many cases are missed and diagnosis is made intraoperatively. ${ }^{2,7)}$ All surgeons are therefore advised to check for signs of great veins involvement as thrombectomy with vascular repair or reconstruction is usually possible.

\section{Conflict of Interest}

This material has never been published and is not currently under evaluation in any other peer- reviewed publication. There is no conflict of interest or financial disclosure to be made.

\section{Funding}

None.

\section{Ethical Approval}

Consent approval from the patient in question has been sought and is readily available upon request.

\section{Orcid}

Roshan Kumar Verma: https://orcid.org/0000-0001-6560-8875 Deepak Sarahan: https://orcid.org/0000-0002-3013-198X Gautamjeet Raj Kumar: https://orcid.org/0000-0003-2551-3091

\section{References}

1) Lang BH, Lo CY, Chan WF, Lam AK, Wan KY. Classical and follicular variant of papillary thyroid carcinoma: a comparative study on clinicopathologic features and long-term outcome. World J Surg 2006;30(5):752-8.

2) Yu XM, Schneider DF, Leverson G, Chen H, Sippel RS. Follicular variant of papillary thyroid carcinoma is a unique clinical entity: a population-based study of 10,740 cases. Thyroid 2013;23(10):1263-8.

3) Chang HY, Lin JD, Chou SC, Chao TC, Hsueh C. Clinical presentations and outcomes of surgical treatment of follicular variant of the papillary thyroid carcinomas. Jpn J Clin Oncol 2006;36(11):688-93.

4) Mete O, Asa SL. Pathological definition and clinical significance of vascular invasion in thyroid carcinomas of follicular epithelial derivation. Mod Pathol 2011;24(12):1545-52.

5) Holt WL Jr. Extension of malignant tumors of thyroid into great veins and heart. JAMA 1934;102(23):1921-24.

6) Graham AR. Malignant epithelial tumors of the thyroid with special reference to invasion of blood vessels. Surg Gynecol Obstet 1924;39:781-90.

7) Gross M, Mintz Y, Maly B, Pinchas R, Muggia-Sullam M. Internal jugular vein tumor thrombus associated with thyroid carcinoma. Ann Otol Rhinol Laryngol 2004;113(9):738-40.

8) Koike E, Yamashita H, Watanabe S, Yamashita H, Noguchi S. Brachiocephalic vein thrombus of papillary thyroid cancer: report of a case. Surg Today 2002;32(1):59-62.

9) Bukhari H, Ayad M, Rosenthal A, Block M, Cortelli M. Superior vena cava syndrome secondary to thyroid cancer. J Surg Case Rep 2011;2011(7):7. 
10) Kobayashi K, Hirokawa M, Yabuta T, Fukushima M, Kihara M, Higashiyama T, et al. Tumor thrombus of thyroid malignancies in veins: importance of detection by ultrasonography. Thyroid 2011;21(5):527-31.

11) Fotis T, Konstantinou E, Mariolis-Sapsakos T, Mitsos A, Restos S, Katsenis K, et al. Solitary internal jugular vein invasion by thyroid carcinoma: resection and reconstruction. $J$
Vasc Nurs 2009;27(2):46-7.

12) Dikici AS, Yildirim O, Er ME, Kilic F, Tutar O, Kantarci $\mathrm{F}$, et al. A rare complication of the thyroid malignancies: jugular vein invasion. Pol J Radiol 2015;80:360-3.

13) Carrington BM, Adams JE. Jugular vein thrombosis associated with distant malignancy. Postgrad Med J 1988;64(752):455-8. 